\title{
NUESTRA PARTICIPACIÓN EN LOS CONGRESOS CIENTÍFICOS NACIONALES DE ESTUDIANTES DE MEDICINA, 1993-2010.
}

\section{OUR PARTICIPATION IN NATIONAL SCIENTIFIC CONGRESS OF MEDICAL STUDENTS, 1993-2010}

\author{
J. Jhonnel Alarco ${ }^{1,2, a, b}$, Esmilsinia V. Álvarez-Andrade ${ }^{1,2, a}$ \\ 1. Facultad de Medicina, Universidad Nacional San Luis Gonzaga. Ica, Perú. \\ 2. Sociedad Científica de Estudiantes de Medicina de Ica. \\ a. Estudiante de Medicina, b. Químico Farmacéutico
}

La Sociedad Científica Médico Estudiantil Peruana (SOCIMEP) es una institución dedicada al fomento de la investigación científica, que congrega a Sociedades Científicas Estudiantiles de 25 Facultades de Medicina de todo el Perú y que cada año organiza el Congreso Científico Nacional (CCN) evento máximo de la investigación a nivel de pregrado en nuestro país en el que se presentan un promedio de 60 investigaciones ${ }^{(1,2)}$.

Estos trabajos científicos son presentados y sometidos a la crítica siendo evaluados tanto por estudiantes participantes así como por un selecto comité de expertos quienes determinan luego de una rigurosa revisión los 10 mejores trabajos (top ten), y por supuesto, el trabajo ganador, que no solo premia el esfuerzo del autor o autores, sino que otorga además un prestigio adicional a la Facultad de Medicina de origen de la investigación.

En nuestra universidad existe la Sociedad Científica de Estudiantes de Medicina de Ica (SOCEMI), encargada de promover el de desarrollo de la investigación entre los estudiantes de medicina de pregrado, institución que ha tenido participación, no solo en la presentación de trabajos originales en los CCN, sino llegando incluso a ocupar los primeros puestos, obteniendo reconocimiento tanto a nivel nacional como internacional, además, sus miembros han ocupado cargos importantes y de gran responsabilidad dentro de la estructura de organización de la SOCIMEP (3).

En el transcurso de 18 años de realizados los CCN, se han presentado un total 1193 trabajos científicos, la SOCEMI ha participado con 95 investigaciones, representando el $8 \%$ del total. En los últimos ocho años (2003-2010) se ha observado un marcado descenso en el número de investigaciones presentadas por la SOCEMI a los CCN pasando de tener un papel protagónico en años anteriores a presentar solo trabajos aislados, cuya realización, en la mayoría de los casos, se realiza sin apoyo de docentes, Figura 1.
La participación estudiantil en este tipo de eventos científicos es importante ya que proporciona experiencias que promueven una actitud positiva hacia la práctica y desarrollo de la investigación científica.

Consideramos que nuestras autoridades universitarias deben comenzar a motivar y facilitar la participación de sus estudiantes, no solo a los CCN, sino también a cursos de redacción y publicación científica, mejorando nuestro protagonismo en eventos científicos nacionales e internacionales, promoviendo a su vez a investigadores que aun siendo estudiantes, podrían incentivar a las nuevas generaciones de estudiantes a investigar y a publicar desde pregrado, creando de esta manera un circulo saludable de investigación científica ${ }^{(4)}$.

Si bien es cierto que la investigación científica que se realiza de manera extracurricular y que no todos los estudiantes y profesionales son participes de ella, la existencia de sociedades científicas estudiantes a las que se vinculan miembros con cualidades de curiosidad por aprender a crear conocimiento desde el pregrado es una oportunidad que debe ser aprovechada por nuestras instituciones $^{(5)}$.

En países del primer mundo universidades invierten en programas para incentivar a los estudiantes de pregrado a la investigación, ya que reconocen que ellos serán el futuro de sus países, sin embargo en países en vías de desarrollo como el nuestro, no necesitamos crear este tipo de programas ya que estos se encuentran conformados, solo hace falta invertir en ellos ${ }^{(6)}$.

\section{Correspondencia:}

Jhonnel Alarco

Correo electrónico: jhonnelalarco@hotmail.com 


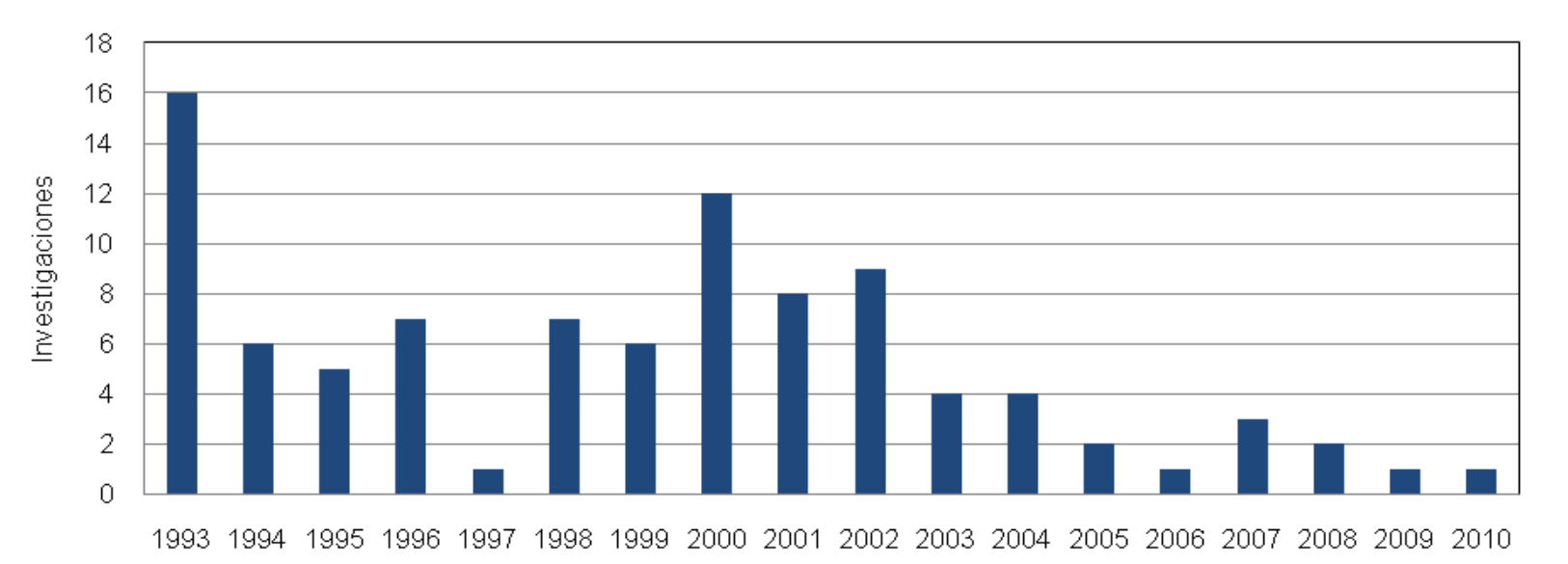

Figura 1. Investigaciones presentadas por la SOCEMI a Congresos Científicos Nacionales de estudiantes de medicina

\section{REFERENCIAS BIBLIOGRÁFICAS}

1. Sociedad Científica Medico Estudiantil Peruana (SOCIMEP) (Sitio en Internet). Disponible en: http://socimep.org/website/index.php?option=com_content\&view=article\&id=46\&ltemid=63. Acceso el 8 de Octubre del 2010.

2. Huamaní C, Chávez-Solís P, Domínguez-Haro W, Solano-Aldana M. Producción científica estudiantil: Análisis y Expectativas. Rev Peru Med Exp Salud Pública. 2007; 24(4):444-46.

3. Galán E, Manrique N, Villavicencio E, Yllatopa E, Peralta M, De la Cruz W. Producción científica de los investigadores del pregrado de medicina humana del Perú, 1993-2003. CIMEL. 2005; 10(1):41-48.

4. Arroyo-Hernández $\mathbf{C H}$, Zukerán-Medina EB, Miranda-Soberón UE. Características de la producción científica biomédica en Ica, Perú 1998-2007. Rev Perú Med Exp Salud Pública. 2009; 26(2):203-206.

5. Arroyo-Hernández $\mathbf{C H}$, De la Cruz W, Miranda-Soberón UE. Dificultades para el desarrollo de investigaciones en pregrado en una universidad pública de provincia, Perú. Rev Peru Med Exp Salud Publica. 2008; 25(4): 348-49.

6. Mayta-Tristán P. Sociedades científicas de estudiantes de medicina: el futuro de la investigación en Latinoamérica. MedUNAB 2010; 13(1): 3-4. 